\title{
Estudantes de medicina dos três primeiros anos são os principais ingressantes na Liga Acadêmica de Medicina de Urgência e Emergência
}

\author{
Medical Students of the first three years are the main entrants in the Urgency \\ and Emergency Medicine Academic League
}

\author{
Mateus de Sousa Rodrigues ${ }^{1}$, Ivan Martins Galvão²
}

Rodrigues MS, Galvão IM. Estudantes de medicina dos três primeiros anos são os principais ingressantes na Liga Acadêmica de Medicina de Urgência e Emergência / Medical students of the first three years are the main entrants in the Urgency and Emergency Medicine Academic League. Rev Med (São Paulo). 2017 jul.-set.;96(3):136-9.

RESUMO: A Liga Acadêmica de Medicina de Urgência e Emergência (LAMURGEM) é uma entidade sem fins lucrativos que estuda as emergências clínicas e cirúrgicas e desenvolve atividades nessa área. No entanto, não existem estudos que abordam o perfil dos ingressantes nessa liga e os fatores que levam os estudantes a realizarem essa escolha. Esse estudo retrata o padrão dos inscritos e ingressantes na LAMURGEM, comparando os resultados obtidos com os achados da literatura. Trata-se de um estudo transversal, realizado a partir da análise dos trinta e oito estudantes inscritos no processo seletivo da LAMURGEM em 2016. Foi analisado o perfil dos estudantes inscritos e ingressantes de acordo com o período cursado. $100 \%$ dos estudantes inscritos estavam entre o segundo e o sexto período do curso de medicina, e o número de inscritos no processo seletivo diminuiu ao longo dos períodos. Esse trabalho mostra que estudantes dos primeiros períodos são os ingressantes majoritários na LAMURGEM, confirmando os resultados abordados nas literaturas.

Descritores: Estudantes de medicina/classificação; Medicina de emergência/educação; Educação médica; Educação de graduação em medicina/tendências.
ABSTRACT: The Urgency and Emergency Medicine Academic League (LAMURGEM) is a nonprofit entity that studies surgical and clinical emergencies and develops activities in this area. However, there aren't any studies that evaluate the profile of entering students in this league and the factors that lead them to this choice. This investigation depicts the pattern of inscribed and entering students in LAMURGEM, comparing the results with other literature findings. This is a retrospective observational study involving the analysis of thirty-eight students inscribed in the selection test for entering in LAMURGEM in 2016. The profile of inscribed and entering students was analyzed according to the graduation period. One hundred percent $(100 \%)$ of the inscribed students were between the second and sixth semester of medical course, and the number of inscribed students in the selection process progressively reduced over the semesters. This paper shows that students of the initial periods are the majority of entering students in LAMURGEM, confirming the results discussed in the literature.

Keywords: Students, medical/classification; Emergency medicine/education; Medical education; Education, medical, undergraduate/trends.

1. Diretor científico da Liga Acadêmica de Medicina de Urgência e Emergência (LAMURGEM) da Universidade Federal do Vale do São Francisco (UNIVASF). Email: mateuserem@gmail.com.

2. Médico de Família e Comunidade, especialista em Dermatologia e docente da Universidade Federal do Vale do São Francisco, Petrolina, PE, Brasil. Email: ivan.galvão@univasf.edu.br.

Endereço para correspondência: Mateus de Sousa Rodrigues. Av. José de Sá Maniçoba, s/n - Centro, Petrolina, PE. CEP: $56304-917$. 


\section{INTRODUÇÃO}

$\Lambda_{\text {sem fins lucrativos que proporcionam aos }}^{\text {s ligas acamicas são entidades universitárias }}$ seus membros atividades didáticas, científicas, culturais e sociais, estudando determinada área da saúde ${ }^{1,2}$. Elas são formadas pelos próprios estudantes sob supervisão e orientação dos docentes ${ }^{3}$. Nas ligas acadêmicas, os discentes desenvolvem seminários, apresentações de casos clínicos e artigos, elaboração de projetos científicos, acompanhamento de plantões e cirurgias e treinamento de procedimentos médicos ${ }^{1}$.

Em 1920, a Liga de Combate à Sífilis foi a primeira liga acadêmica fundada no Brasil. Essa Liga está vinculada à Faculdade de Medicina da Universidade de São Paulo e existe até hoje. Isso reflete a longevidade que essas atividades atingem ${ }^{1}$. Houve um aumento expressivo do número de ligas durante a ditadura militar ${ }^{4}$. Entretanto, não havia extensão das atividades para a comunidade. $\mathrm{Na}$ primeira década do presente século, a quantidade de ligas se intensificou coincidindo com o período de importantes reformas curriculares ${ }^{1}$. Em 2006, surgiu a Associação Brasileira de Ligas Acadêmicas de Medicina, que foi a maior conquista dessas atividades no país.

A Liga Acadêmica de Medicina de Urgência e Emergência (LAMURGEM) foi a primeira liga criada na Universidade Federal a qual ela está vinculada. Ela foi fundada em março de 2008, e é filiada a Associação Brasileira de Urgência e Emergência (ABRAMURGEM) e a Sociedade Brasileira de Clínica Médica. Na LAMURGEM, as atividades desenvolvidas são: apresentação de capítulos de livros de emergência e artigos científicos, acompanhamento de plantões em pronto-socorro e atendimento extra-hospitalar, realização de sessão aberta e simpósios, extensão sobre temas relacionados a primeiros socorros e prevenção de acidentes. O ingresso na liga é permitido a partir do segundo período mediante a realização do processo seletivo que ocorre anualmente.

Estudos demonstram que as ligas acadêmicas proporcionam benefícios e riscos à formação médica. Paulo et al. ${ }^{1}$ apontam que os pontos positivos dessas atividades são preponderantes. Elas são uma chance adicional para a aprendizagem e permitem que os estudantes sejam protagonistas de suas atividades ${ }^{1}$. A participação em ligas acadêmicas é importante, desde que não interfira no desenvolvimento dos componentes do currículo formal ${ }^{5}$. Os principais problemas dessa atividade apontados pela literatura são: ocupam o tempo livre abreviado dos discentes; podem estimular o exercício ilegal da medicina quando na ausência do docente; podem permitir a aprendizagem de conceitos distorcidos ou condutas antiéticas; podem se tornar sociedades científicas desprovidas de promoção de saúde; influenciam à especialização precoce; estimulam a competitividade dos estudantes pelos programas de residência ${ }^{4,5}$.

Esse trabalho objetiva demonstrar o perfil dos inscritos e ingressantes na LAMURGEM, comparando os resultados obtidos com os achados da literatura.

\section{MÉTODOS}

Trata-se de um estudo transversal realizado a partir da análise dos trinta e oito inscritos no processo seletivo da LAMURGEM em 2016. Esses inscritos concorreram às dez vagas disponibilizadas nesse ano mediante a aplicação de uma prova teórica, análise de currículo, apresentação de artigo e entrevista. Apenas estudantes de medicina a partir do segundo período podiam concorrer às vagas.

$\mathrm{O}$ perfil dos estudantes inscritos e ingressantes foi analisado de acordo com o período cursado. Além disso, observou-se o índice de aprovação no processo seletivo por período. Vários artigos científicos foram selecionados no sentido de comparar os resultados com estudos análogos realizados em outras universidades e com achados já bem estabelecidos pela literatura.

\section{RESULTADOS}

O número de inscritos no processo seletivo tende a diminuir ao longo dos períodos, como mostra a Figura 1. O segundo e terceiro período responderam juntos por $65 \%$ das inscrições. Quando analisados isoladamente, aquele correspondeu a $42 \%$ enquanto esse atingiu $23 \%$. O quarto, quinto e o sexto período corresponderam a $16 \%, 13 \%$ e $6 \%$, respectivamente. O sétimo e o oitavo períodos não tiveram nenhum participante.

Apenas os períodos pares representados na Figura 1 mantiveram uma proporção quase constante entre o número de inscritos e ingressantes. O segundo período respondeu por $40 \%$ das vagas disponibilizadas e $25 \%$ dos seus inscritos ingressaram na liga. 30\% dos ingressantes eram do quarto período. A proporção entre aprovados e inscritos nesse período foi $50 \%$. O sexto período correspondeu a $20 \%$ das aprovações. Analogamente ao quarto período, a proporção entre ingressantes e inscritos desse grupo foi de $50 \%$.

Os períodos ímpares representados na Figura 1 apresentaram um baixo índice de aprovados. O terceiro e o quinto períodos responderam por $10 \%$ dos ingressantes cada um. Apenas $11 \%$ dos inscritos do terceiro período conseguiram ingressar na liga. No quinto período, apenas $20 \%$ dos inscritos obtiveram êxito no ingresso. 


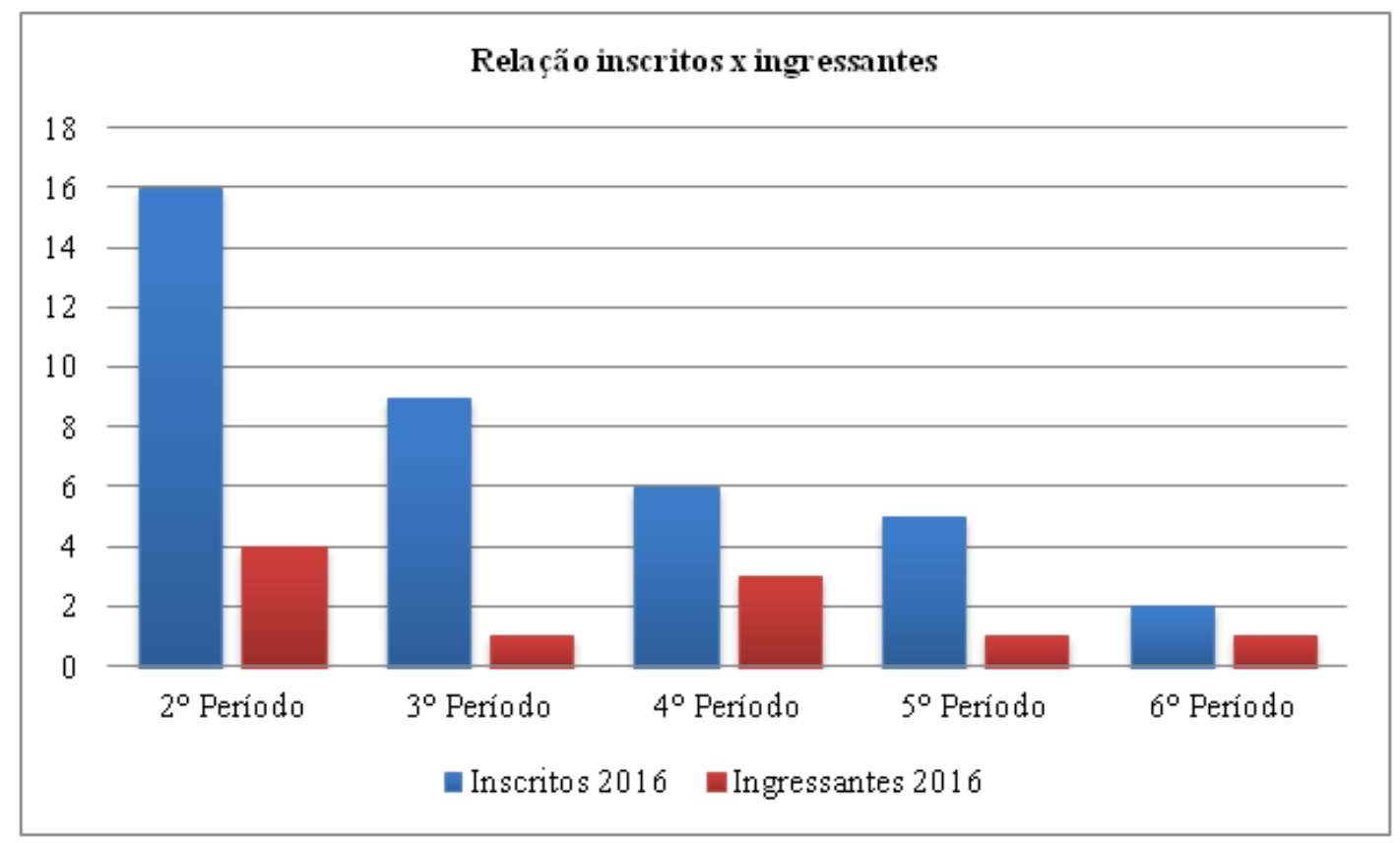

Figura 1. Inscritos e ingressantes na LAMURGEM em 2016 por período cursado

\section{DISCUSSÃO}

Estudantes pertencentes aos períodos pares obtiveram um rendimento superior aos discentes de períodos ímpares no processo seletivo da LAMURGEM. Isso pode ser atribuído a maior complexidade e menor disponibilidade de tempo livre dos períodos ímpares na universidade, sobretudo no caso de estudantes do quinto período. Esse é o período mais complexo do curso de Medicina da universidade pelos motivos supracitados. Além disso, existem particularidades inerentes a cada turma que contribuíram para esse padrão. Alguns estudantes podem ter realizado o processo seletivo desprovidos de preparação em termos de conteúdo teórico. Outros realizaram o processo seletivo no sentido de testar seus conhecimentos sem objetivo de ingressar na liga.

$O$ ingresso de discentes na LAMURGEM por período é maior entre os primeiros períodos. Todos os inscritos estão entre o primeiro e o terceiro ano do curso. Isso pode estar associado ao fato de que à medida que se avança no curso, o estudante vivencia práticas clínicas no currículo, sem necessariamente pertencer a uma liga. Isso ocorre principalmente a partir do quinto período. Peres et al. ${ }^{6}$ afirmam que entre 70 e $80 \%$ dos estudantes até o quarto ano do curso médico participam de ligas acadêmicas. Mas, esse número reduz após o internato ${ }^{6}$. Essa participação acentuada dos estudantes novatos ocorre pelo desejo de aproximação com a prática médica ainda no início do curso $^{7}$. Estudantes após o internato não possuem esse desejo acentuado, já que estão na fase em que o contato com a prática clínica é diário ${ }^{8}$. Nos inscritos e ingressantes da LAMURGEM, observou-se esse padrão em que a totalidade dos inscritos foi de estudantes até o sexto período. Além disso, os veteranos exercem uma forte influência sobre os calouros a participarem das ligas. No entanto, os estudantes novatos geralmente estão na fase de maior vulnerabilidade emocional dentro da universidade. Assim, eles podem realizar essa decisão sem refletir sobre sua necessidade ${ }^{6}$.

Estudantes iniciantes tendem a ingressar nas ligas também para satisfazer a necessidade psicossocial de serem reconhecidos como médicos. Peres et al. ${ }^{6}$ afirmam que essa atividade é a que mais contempla essa necessidade nos primeiros períodos. $\mathrm{O}$ desejo pela prática de habilidades médicas precocemente tende a estar relacionado com a busca pelo reconhecimento social ${ }^{8}$. Além disso, muitos estudantes participam das práticas hospitalares da liga desprovidos do embasamento de conteúdo teórico que lhes permita compreender os procedimentos realizados pelos médicos. Apesar disso, calouros geralmente acompanham os plantões uma vez que estes lhes permitem vivenciar a experiência de ser médico ${ }^{6}$. Isso pode reduzir as taxas de desistência do curso. Porém, não é produtivo nessa fase.

$\mathrm{O}$ ingresso nas ligas geralmente reflete insuficiências curriculares, a busca por um currículo melhor para os programas de residência e estratégias de socialização. A inadequação do currículo com o mercado de trabalho e o desejo de aumentar a experiência clínica são os principais motivos que levam estudantes a participarem das Ligas acadêmicas ${ }^{9}$. A carência de aulas práticas estabelecidas pelo currículo formal é outro fator que leva os estudantes a ingressarem nas ligas, sobretudo no ciclo básico. Pois nessa fase do curso, o estudante é apenas um espectador na maioria das universidades ${ }^{10}$. Apesar das mudanças curriculares recentes, não houve uma redução do número 
de ligas acadêmicas. Além disso, Peres et al. ${ }^{6}$ afirmam que muitos estudantes vêem a liga acadêmica como resposta para as escolhas da futura especialidade, estratégia de socialização e mecanismo de escape do estresse. Vieira et al. ${ }^{11}$ também defendem que as ligas acadêmicas estimulam a socialização dos estudantes e combatem fatores estressantes do curso. A preparação para provas de residência médica é outro fator que leva muitos acadêmicos a ingressarem nas ligas $^{5}$. Um estudo demonstrou que $62 \%$ dos ligantes da Universidade Federal de Minas Gerais participavam dessa atividade visando à residência médica ${ }^{9}$. Muitos graduandos pensam que a participação em ligas aumenta as chances de ingresso em residências médicas ${ }^{8}$. No entanto, já se sabe que esse aumento é mínimo ${ }^{12}$.

\section{REFERÊNCIAS}

1. Paulo MPF, Alessandro WM. O ensino médico além da graduação: ligas acadêmicas. Diagn Tratamento. 2011;16(2):50-1. Disponível em: http://files.bvs.br/ upload/S/1413-9979/2011/v16n2/a2048.pdf.

2. Neves FBCS, Vieira PS, Cravo EA, et al. Inquérito nacional sobre as ligas acadêmicas de Medicina Intensiva. Rev Bras Ter Intens. 2008;20(1):43-8. doi: 10.1590/S0103507X2008000100007.

3. Monteiro LLF, et al. Ligas acadêmicas: o que há de positivo? Experiência de implantação da Liga Baiana de Cirurgia Plástica. Rev Bras Cir Plast. 2008;23(3):158-60. Disponível em: http://www.rbcp.org.br/details/405/ligas-academicas-o-que-ha-de-positivo--experiencia-de-implantacao-da-ligabaiana-de-cirurgia-plastica.

4. Santana ACDA. Ligas acadêmicas estudantis: o mérito e a realidade. Medicina (Ribeirão Preto). 2012;45(1):96-8. Disponível em: http://revista.fmrp.usp.br/2012/vol45n1/ PV_Ligas\%20Acad\%EAmicas\%20Estudantis.pdf.

5. Pedro THF, Paulo JFV, Fabiane GC, Gabriela OCM, Michelle Zaba, Vinicius CV, Silvana AS. Normatização da abertura de ligas acadêmicas: a experiência da Faculdade de Medicina de Botucatu. Rev Bras Educ Med. 2010;34(1):160-7. doi: 10.1590/S0100-55022010000100019.

6. Peres CM, Andrade AS, Garcia SB. Atividades extracurriculares: multiplicidade e diferenciação necessárias ao currículo. Rev Bras Educ Med. 2007;31(3):203-11. doi: 10.1590/S0100-55022007000300002.

\section{CONCLUSÃO}

Esse trabalho mostrou que estudantes dos primeiros períodos são os ingressantes majoritários na LAMURGEM, confirmando os resultados encontrados na literatura. Além disso, foram discutidos os principais fatores que podem contribuir para esse padrão. Tomando esses fatores em consideração, o estudo salienta a importância de uma reavaliação das reformas curriculares efetivamente adotadas. Além disso, traz conceitos que podem auxiliar os estudantes de medicina a compreender o valor atribuído pelos programas de residência médica à participação nas ligas acadêmicas.

7. Pedro THF. Ligas acadêmicas: motivações e críticas a propósito de um repensar necessário. Rev Bras Educ Med. 2011;535-43. doi: 10.1590/S0100-55022011000400013.

8. Nogueira-Martins MCF, Nogueira-Martins LA, Turato ER. Medical students' perceptions of their learning about the doctor-patient relationship: a qualitative study. Med Educ. 2006;40:322-8. doi: 10.1111/j.1365-2929.2006.02411.x.

9. Tavares AP, Ferreira RA, França EB, Fonseca Júnior CA, Lopes GC, Dantas NGT, et al. O "currículo paralelo" dos estudantes de medicina da Universidade Federal de Minas Gerais. Rev Bras Educ Med. 2007;31(3):254-65. doi: 10.1590/S0100-55022007000300008.

10. Rego S. Currículo paralelo em medicina, experiência clínica e PBL: uma luz no fim do túnel? Interface Comunic Saúde Educ. 1998;2(3):35-48. doi: 10.1590/S141432831998000200004.

11. Vieira EM, Barbieri CLA, Vilela DB, Ianhez Júnior E, Tomé FS, Woida FM, et al. O que eles fazem depois da aula? As atividades extracurriculares dos alunos de ciências médicas da FMRP-USP. Medicina (Ribeirão Preto). 2004;37:8490. Disponível em: http://revista.fmrp.usp.br/2004/ vol37n1e2/5artorig_o_que_eles_fazem_depois_aula.pdf.

12. Huylmer LC, Lucas BB, Daniel CG, Luciano PGC. Vagas para Residência Médica no Brasil: Onde Estão e o que É Avaliado. Rev Bras Educ Méd. 2013;37(4):557-65. doi: 10.1590/S0100-55022013000400011. 